\section{EXPLORING THE IMPACT OF IMMUNOTHERAPY ON CANCER PATIENTS' REAL WORLD EXPERIENCE}

${ }^{1}$ Helen Nichols, ${ }^{1}$ Cynthia Schwartz*, ${ }^{2}$ Elise Wu. ${ }^{1}$ Cancer Support Community, Philadelphia, PA, United States; ${ }^{2}$ Merck \& Co., Kenilworth, NJ, United States

Background Background: Immunotherapy (IO) have demonstrated the superior clinical benefits compared to previous anticancer treatments. A better understanding of the IO patient experience in real world will allow healthcare professionals to develop effective supportive resources, help improve clinical practice and assist patients in managing anticancer journey. However, little information of informational and supportive needs to IO patients are available. This study aims to describe IO cancer patient experiences from multiple perspectives.

Methods This cross-sectional qualitative study consisted of cancer patients, who reported receiving IO targeting PD1/L-1 receptors irrespective of cancer types. The study included three independent focus groups, participants were assigned to a group based on how long they received treatment prior to enrolling in the study: Focus Group 1 (0-6 months), Focus Group 2 (6-12 months), and Focus Group 3 (12+ months), with 4-8 participants each. Thematic analyses followed a deductive approach. Codes and themes were generated based on the European Organization for Research and Treatment of Cancer Quality of Life Questionnaire (EORTC QLQ-C30). ${ }^{1}$ This study was approved by Ethical \& Independent Review Services.

Results: This study included 18 total participants, across three focus groups, diagnosed with cancer from 2006-2020. Fifteen out of 18 were Caucasian; average age was 57.3 years. Participants in all groups reported fatigue as the most common side effect, less ability to do daily tasks due to fatigue, that other patients with the same diagnosis provided the greatest emotional support, concerns regarding finances/careers, and most reported they had adjusted to their "new normal" and were satisfied with the coordination between members of their healthcare team and communication with their oncologist. Participants in Group 1 were more concerned with recurrence and trying to figure out their new roles at home and at work. Participants in Focus Groups 2 and 3 were mostly unable to work due to diagnosis/treatment and reported this was a difficult adjustment that affected their identity. Focus Group 3 participants were particularly concerned about nonmedical expenses and reported receiving insufficient information about IO.

Conclusion: By understanding the IO cancer patient experience at different time points in treatment, healthcare providers can more accurately address unmet needs from patients' perspective, increase healthcare professionals' awareness of IO patients' challenges, and support good communication practices. This will aid healthcare professionals working towards the goal of improving patient well-being, ultimately contributing to treatment adherence in this population.

\section{REFERENCE}

1. Giesinger JM, Kuijpers W, Young $T$, Tomaszewski KA, Friend $E$, Zabernigg $A$, Holzner B, Aaronson NK. Thresholds for clinical importance for four key domains of the EORTC QLQ-C30: physical functioning, emotional functioning, fatigue and pain. Health and Qual Life Outcomes 2016:14:1-8.

Ethics Approval: IRB approval received from Ethical \& Independent Review Services. 